\title{
Nuclear Genomic Sequences Reveal that Polar Bears Are an Old and Distinct Bear Lineage
}

Frank Hailer, ${ }^{1 *}$ Verena E. Kutschera, ${ }^{1}$ Björn M. Hallström, ${ }^{1}$ Denise Klassert, ${ }^{1}$ Steven R. Fain, ${ }^{2}$ Jennifer A. Leonard, ${ }^{3}$ Ulfur Arnason, ${ }^{4}$ Axel Janke ${ }^{1,5 *}$

Recent studies have shown that the polar bear matriline (mitochondrial DNA) evolved from a brown bear lineage since the late Pleistocene, potentially indicating rapid speciation and adaption to arctic conditions. Here, we present a high-resolution data set from multiple independent loci across the nuclear genomes of a broad sample of polar, brown, and black bears. Bayesian coalescent analyses place polar bears outside the brown bear clade and date the divergence much earlier, in the middle Pleistocene, about 600 (338 to 934) thousand years ago. This provides more time for polar bear evolution and confirms previous suggestions that polar bears carry introgressed brown bear mitochondrial DNA due to past hybridization. Our results highlight that multilocus genomic analyses are crucial for an accurate understanding of evolutionary history.

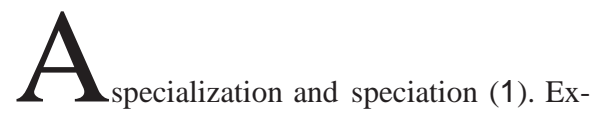

found in nature, providing a route to novel sequence space. The capacity of synthetic polymers for both

RNA are not functionally unique as genetic materials. The methodologies developed herein are readily applied to other nucleic acid architectures and have the potential to enable the replication of genetic polymers of increasingly divergent chemistry, structural motifs, and physicochemical properties, as shown here by the acid resistance of HNA aptamers (fig. S17). Thus, aspects of the correlations between chemical structure, evolvability, and phenotypic diversity may become amenable to systematic study. daptation to novel environmental conditions is an important driver of niche

${ }^{1}$ Biodiversity and Climate Research Centre (BiK-F), Senckenberg Gesellschaft für Naturforschung, Senckenberganlage 25, 60325 Frankfurt am Main, Germany. ${ }^{2}$ National Fish and Wildlife Forensic Laboratory, 1490 East Main Street, Ashland, OR, USA. ${ }^{3}$ Conservation and Evolutionary Genetics Group, Estación Biológica de Doñana (EBD-CSIC), Avenida Américo Vespucio, s/n, 41092 Seville, Spain. ${ }^{4}$ Lund University Hospital, Box 117, 22100 Lund, Sweden. ${ }^{5}$ Goethe University Frankfurt, Institute for Ecology, Evolution and Diversity, 60438 Frankfurt am Main, Germany.

*To whom correspondence should be addressed. E-mail: frashai@gmx.net (F.H.); ajanke@senckenberg.de (A.J.) cept for special cases such as hybrid speciation (2), the speciation process is generally considered to be rather slow in mammals: $\mathrm{Pa}$ leontological and genetic evidence indicate that most species pairs or sister lineages of mammals diverged at least 1 million years ago $(3,4)$. One notable exception seems to be the polar bear (Ursus maritimus), a uniquely adapted high-arctic specialist $(5,6)$ for which recent studies have suggested a surprisingly modern matrilineal origin at less than 111 to 166 thousand years ago (ka) (7-9). These studies found extant polar bears rooted 
A Nuclear DNA

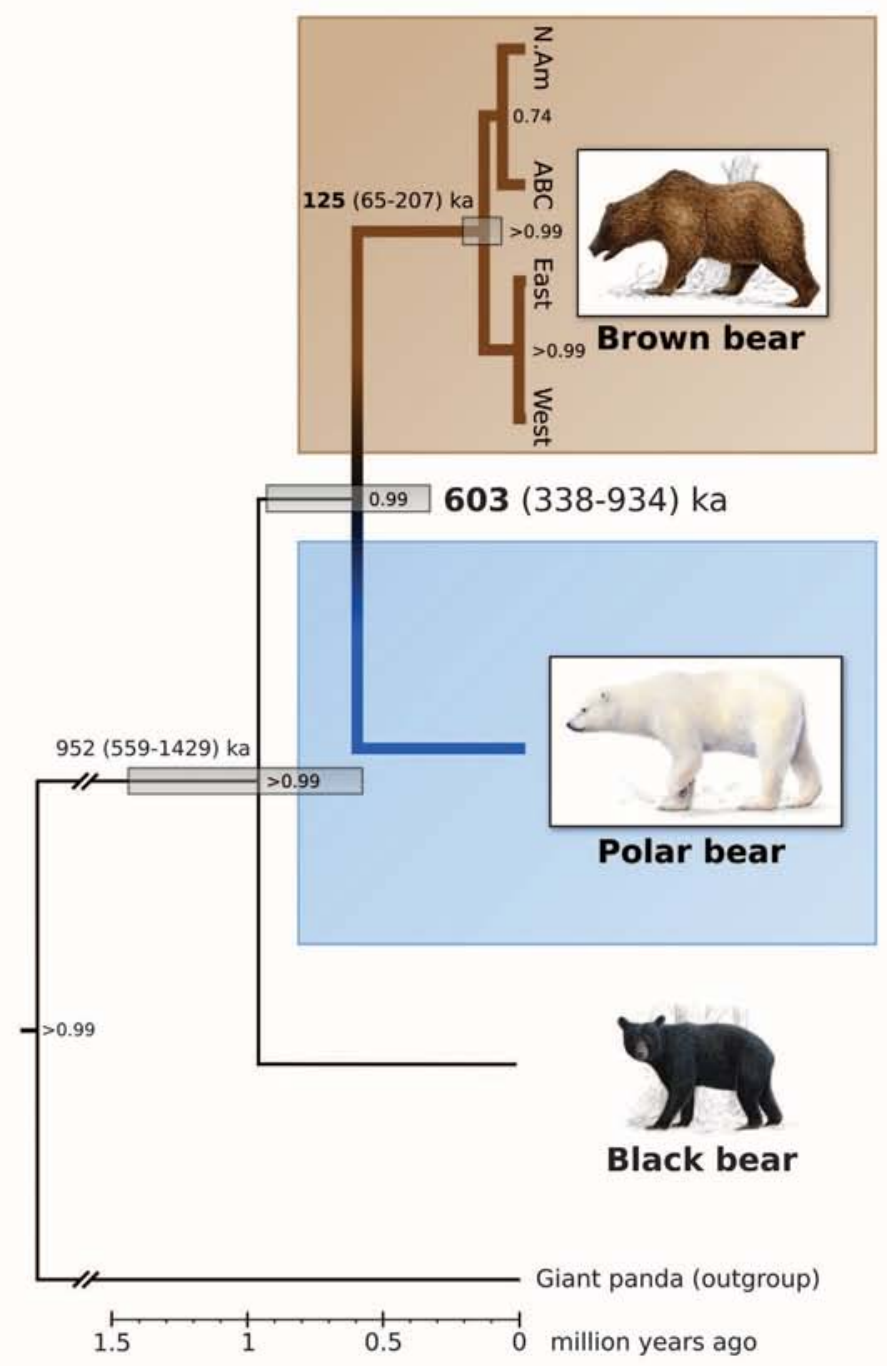

C

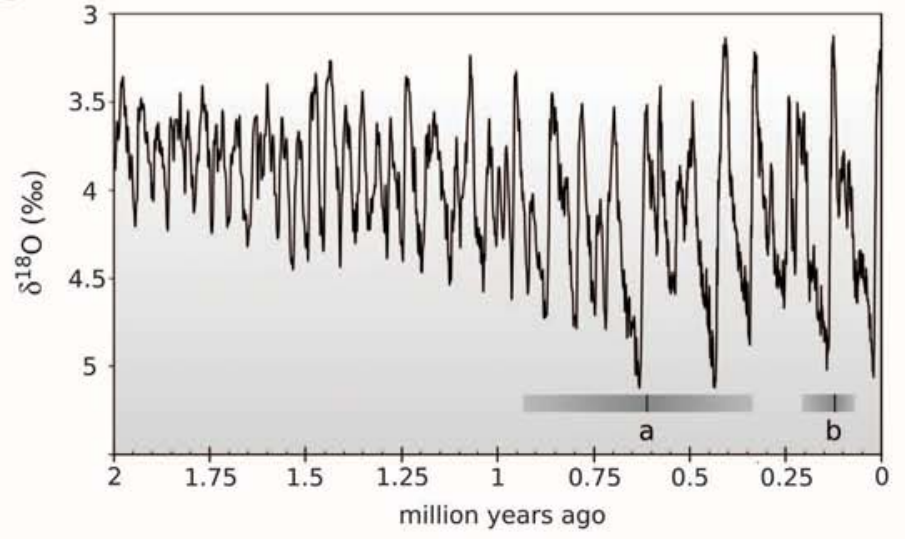

B Mitochondrial DNA
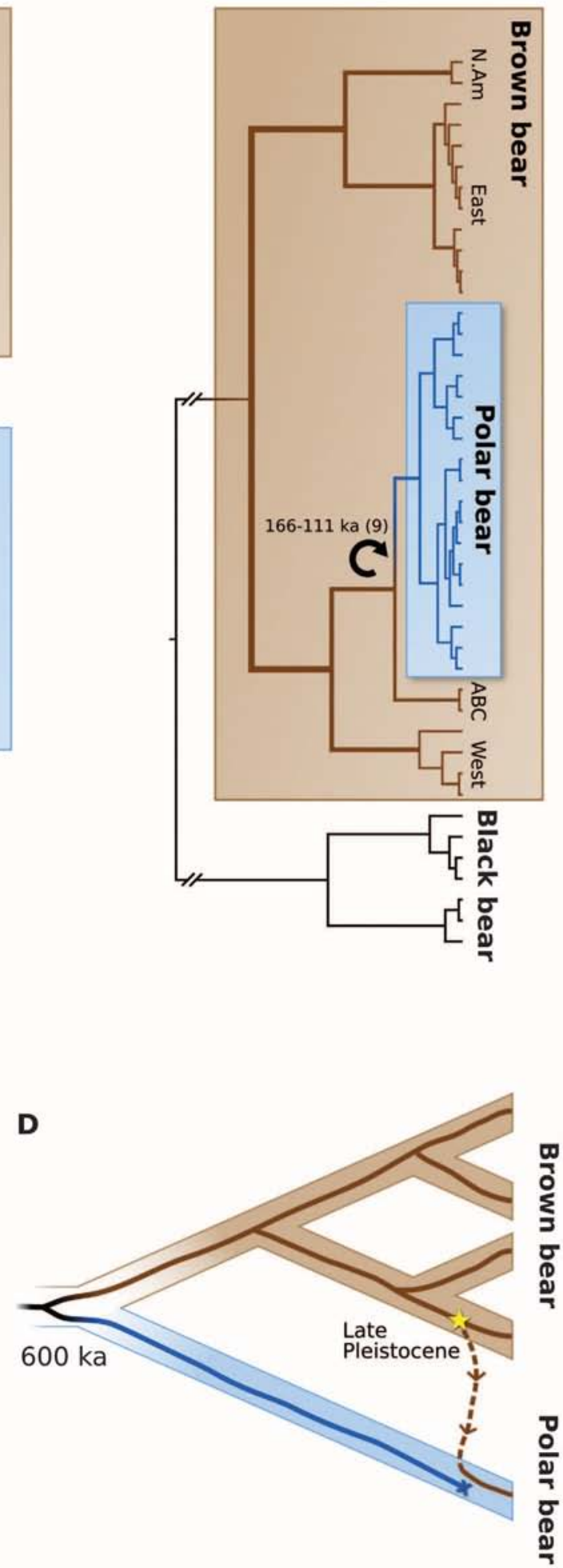

Fig. 1. Ancient origin of polar bears and subsequent introgressive replacement of their mitochondrial DNA with a brown bear haplotype. (A) Species tree of nuclear intron data. Polar and brown bears are sister groups, with their divergence time estimated at 603 (338 to 934) ka. Numbers next to nodes indicate statistical support, and gray bars are 95\% highest credibility ranges for node ages. (B) MtDNA phylogeny. Polar bears are nested within the brown bear clade. The circular arrow denotes mtDNA replacement in polar bears before 166 to 111 ka [upper and lower 95\% confidence limits from (9)]. Clades are named according to previously identified mtDNA lineages in brown bears $(8,18)$. (C) Temperature curve since the Pleistocene [modified from (32)], and evolutionary events in bears. "a" denotes the origination of the polar bear lineage and " $\mathrm{b}$ " the diversification of extant brown bear lineages. Shaded gray bars are 95\% credibility intervals; black lines denote median estimates. (D) Schematic scenario for mtDNA inheritance in bears. Speciation occurred in the middle Pleistocene, but hybridization during the late Pleistocene led to mtDNA similarity between extant polar bears and brown bears from the $\mathrm{ABC}$ islands $(7,8)$ and Ireland (9). The star denotes the brown bear ancestor of extant polar bear mtDNA and the " $X$ " a hypothesized disappearance of the ancestral matriline in polar bears. mtDNA data from ancient remains indicate additional instances of hybridization (9). 
within brown bear (U. arctos) diversity, as a sister lineage to brown bears from the Alaskan Admiralty, Baranof, and Chichagof (ABC) islands or Ireland (7-9). Those results would render brown bears paraphyletic and are consistent with the absence of polar bear fossils before the late Pleistocene (7-10).

To date, the evolutionary history of polar and brown bears has primarily been studied using mitochondrial DNA (mtDNA) (7-9, 11). The few studies that employed nuclear markers included only single representations per species, therefore lacking power to assess paraphyly $(9,12-14)$. Although mtDNA analyses are routinely used in phylogenetics and phylogeography, they have well-known limitations, especially in cases of sex bias and/or introgressive hybridization (15). Its maternal inheritance renders mtDNA sensitive to random genetic drift but insensitive to male-biased gene flow. Moreover, its inheritance as a single linked molecule prevents the estimation of genome-wide population genetic parameters (16). To test the mtDNA-based observation of brown bear paraphyly (some brown bears being more closely related to polar bears than to some conspecifics), nuclear sequences from a diversity of brown and polar bears are required. Studies using multiple unlinked nuclear loci from a broad population sample allow the analysis of autonomously inherited genetic markers. These yield statistically independent information and are therefore essential for recovering an unbiased picture of evolutionary relationships (e.g., species trees) and for obtaining accurate estimates of divergence times (16).

Table 1. Mitochondrial and nuclear genetic diversity in bears. $n$, number of analyzed individuals; $p$, Tamura-Nei corrected nucleotide diversity.

\begin{tabular}{lrcc} 
Species & $\mathrm{n}$ & $\begin{array}{c}\mathrm{p}(\mathrm{mtDNA}) \\
\left(\times 10^{-3}\right)\end{array}$ & $\begin{array}{c}\mathrm{p}(\text { nuclear DNA }) \\
\left(\times 10^{-3}\right)\end{array}$ \\
\hline Polar & 19 & 5.45 & 0.575 \\
Brown & 18 & 25.13 & 2.496 \\
Black & 7 & 17.75 & 1.437
\end{tabular}

We sequenced and analyzed 9116 nucleotides from 14 independent nuclear loci (introns) across the genome in 45 individuals of polar, brown, and black bears (tables S1 and S2) (17), using the giant panda as an outgroup (17). A species tree was reconstructed using a Bayesian multilocus coalescent approach (Fig. 1A) (16) that embeds the gene trees for each locus in a separately estimated species tree. With high statistical support $(\mathrm{P}>0.99)$, polar bears were recovered as a sister lineage to all brown bears, and their divergence time was estimated at 603 ka (median estimate), with 95\% credibility intervals (338 to $934 \mathrm{ka}$ ) that exclude the time frame determined for the extant matriline (111 to $166 \mathrm{ka}$ ) (9). A phylogenetic analysis of the concatenated data (fig. S2) and a neighbor-joining tree of pairwise differentiation estimates $\left(F_{\mathrm{ST}}\right)$ (fig. S3) confirmed this multilocus analysis. Our results thus provide a fundamentally different picture of the polar bear's evolutionary history, compared with the "recent-origin" scenario suggested for its mtDNA $(7,8)$.

Due to the contrasting evolutionary scenarios provided by our nuclear versus published mtDNA data, we analyzed (17) a 640-base pair section of the mitochondrial control region to verify that our samples represent the main lineages of extant brown and polar bears and that their evolutionary relationships reflect the current view of bear mtDNA phylogeny. Indeed, following the nomenclature of Leonard et al. (18), our sampling covers brown bear clades $1,2,3$, and 4 , encompassing individuals from northern and central Europe as well as the Alaskan ABC islands and across continental North America (Fig. 1B). Consistent with previous mtDNA studies (7-9, 11), all polar bears clustered together with high posterior node support $(P>0.99)$ within the diversity of brown bears, sharing a most recent common ancestor with the $\mathrm{ABC}$ island lineage (clade 2a). Our sampling includes two of the most strongly differentiated microsatellite clusters in polar bears (19) (east versus west Greenland), as well as continental North America and Iceland (17). There- fore, this study represents a high-resolution data set that compares nuclear genomic variation in multiple polar and brown bear individuals, providing an independent view of their evolutionary history.

Recently diverged species still share many alleles in their nuclear genomes because of retained ancestral polymorphisms $(20,21)$. Therefore, given the recent mtDNA divergence among extant polar and brown bears, one might expect the two species to share a majority of nuclear haplotypes. However, numerous nuclear haplotypes were unique to polar bears. Across all polar and brown bear samples, we encountered a total of 114 haplotypes at the 14 intron loci (table S3). Out of 35 haplotypes in polar bears and 79 in brown bears, only 6 were shared (table S3 and fig. S1), and most of these were rare in at least one taxon. For the majority of nuclear loci, polar bear sequences were distinct from those in brown bears (fig. S1), and at least 20 sites were fixed. Nucleotide diversity in polar bears was only about $20 \%$ of that in brown bears (Table 1), with 22 single-nucleotide polymorphisms in polar bears and 95 in brown bears. These analyses support that polar bears are a distinct and genetically differentiated species, rather than a lineage that evolved recently from a brown bear genotype. Although the polar bear genome thus harbors an unexpected abundance of unique genetic variation, effective population size is lower than that of its southern relative. This commonly observed biogeographic pattern likely reflects smaller long-term population sizes and stronger population bottlenecks in arctic than in temperate species (22).

Overall nuclear genomic differentiation (multilocus $\mathrm{F}_{\mathrm{ST}}$ ) between polar and brown bears (0.692) was similar to that between each of these and black bears (brown-black 0.685, polar-black 0.893) (table S4), consistent with long-term genetic distinctiveness of polar bears. These findings agree with the nuclear species tree (Fig. 1A) but differ from the matrilineal scenario. A recent estimate of the polar/brown bear divergence time ( 0.4 to 2 million years)
Fig. 2. Individual-based clustering results from nuclear variation in bears. Vertical bars show the cluster membership of each individual, for a clustering into five groups (indicated by separate colors). Polar bears (blue) appear to be genetically more homogeneous than brown bears, within which a subclustering is discernible (light and dark brown). Note the absence of multilocus introgression signals among brown and polar bears, indicating that much of the polar bear genome is unaffected by (recent) hybridization.

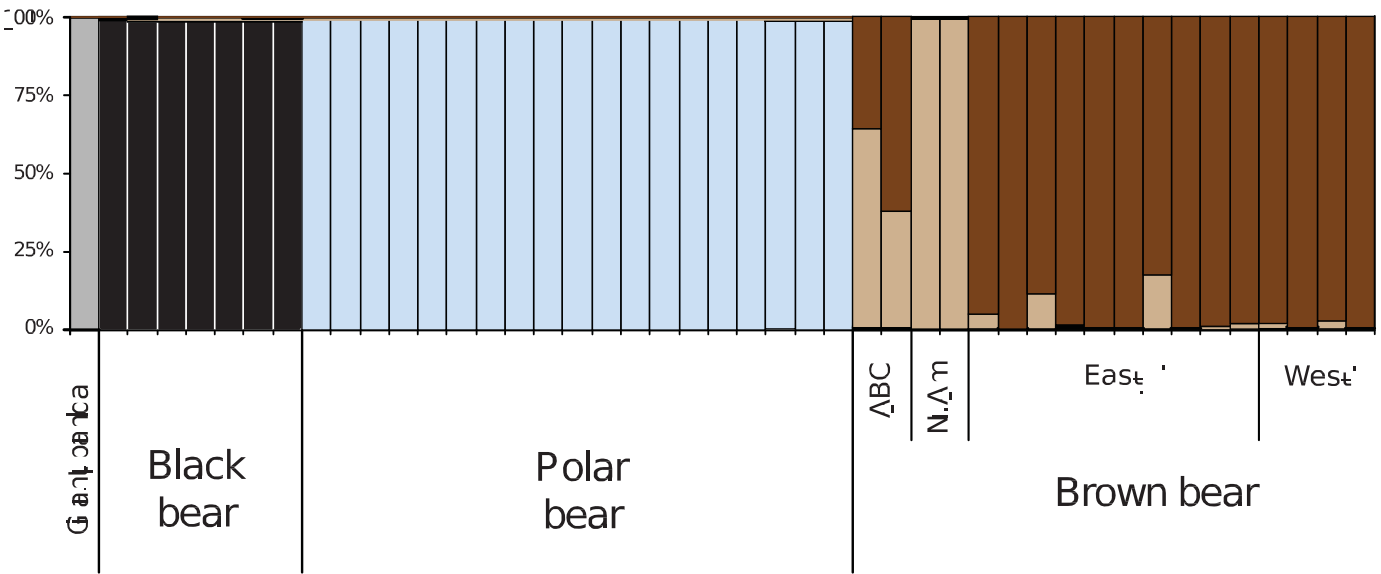


based on nuclear loci and single representations per species supports our results (9). Previous studies provided an older time frame for the black/brown bear divergence $(7,17)$ than our data (Fig. 1A). Therefore, our dating of the polar/brown bear divergence could prove an underestimate, which would strengthen our main conclusions. Our nuclear dating closely resembles the time frame for speciation of another high-arctic specialist, the arctic fox, which diverged from its sister lineage about 900 ka (23). In accordance with matrilineal data (9), our nuclear gene analyses place the origin of extant brown bear diversity at approximately 125 (65 to 207) ka. The warm Eemian interglacial period at $125 \mathrm{ka}$ and the ensuing onset of late Pleistocene glacial cycles (Fig. 1C) may explain the fragmentation and origin of modern brown bear lineages. Extant genetic diversity in the gray wolf also dates back to this time (24). Our dating of evolutionary events in bears therefore coincides with analogous events in other Eurasian and North American carnivores. Kurtén's allometry-based suggestion (10) that polar bears could have evolved during the middle Pleistocene supports our finding, although the oldest known polar bear fossils date to less than 110 to $130 \mathrm{ka}$ (7-10). The life of polar bears on coastal ice, an ephemeral habitat shaped by multiple glacial advances and retreats, may explain why older fossils have not been found.

An earlier evolutionary origin of the polar bear lineage requires a reinterpretation of the established branching pattern of mtDNA lineages $(7-9,11)$. In principle, all extant brown and polar bear mtDNA could be of polar bear origin, but this would require several events of hybridization and subsequent mtDNA replacement (9), which appears unlikely in a widespread generalist species like the brown bear. More parsimoniously, introgressed brown bear material may have replaced the original polar bear mtDNA (Fig. 1D). This would imply that female brown bears mated with male polar bears and that the offspring backcrossed into the polar bear population [consistent with recent observations of fertile hybrids in the wild (25)]. Polar and brown bears are not generally codistributed, but polar bears colonizing coastal land due to sea ice melting during the last interglacial could have been susceptible to introgression from resident brown bears $(25,26)$. Regardless of the direction of mtDNA replacement, such a process has been described for other mammals $(27,28)$.

A Bayesian multilocus genotype clustering analysis $(17,29)$ revealed no detectable signal of recent or ongoing nuclear gene flow between the polar and brown bear individuals (Fig. 2 and fig. S5). Similarly, migration rates among polar and brown bear groups did not differ significantly from zero, as shown by coalescent-based multilocus simulations (table S5 and fig. S4) (30). This suggests that polar/brown bear hybridization is currently infrequent and/or limited to a few geographic regions (25). Nevertheless, mtDNA yields a signal of at least one or two hybridization events during the late Pleistocene (9), illustrating the usefulness of haploid, uniparentally inherited loci and ancient DNA studies to track reticulate evolutionary relationships (7-9). To obtain the overall species tree and associated timing estimates, however, the use of multiple independent loci is crucial. This study therefore highlights that mtDNA does not always reflect the species' overall (genomewide) evolutionary history.

Despite the lack of average, multilocus signals of frequent or recent bear hybridization (Fig. 2 and fig. S4), indications of admixture are not limited to mtDNA. Some loci can remain informative about past hybridization events even when most of the genome is unaffected (31). We found a candidate locus for introgression by polar/brown bear hybridization. At the intron locus 11080, bears clustered in species-specific haplogroups (fig. S1), with one notable outlier: ABC island brown bears carried the (fixed) polar bear haplotype. If this pattern reflects introgression, it could represent evidence of polar bear genetic material in brown bears, suggesting gene flow in the opposite direction relative to mtDNA (9). Because the process of lineage sorting is slow, spanning time scales relevant to speciation $(20,21)$, linkage mapping studies like those in canids (31) will be necessary to pinpoint the phylogenetic origins of individual alleles in bears. Adaptive introgression not only may have helped polar bears to withstand interglacial warm phases (9) and potentially counteracted inbreeding but also may have facilitated the persistence of brown bear populations in subarctic landscapes.

In conclusion, our data suggest that polar bears are a genetically distinct lineage that is older than previously recognized. An evolutionary origin several hundred thousand years ago implies that polar bears as a species have experienced multiple glacial cycles and have had considerable time to adapt to arctic conditions. However, the low genetic diversity in polar bears suggests that changes in the environment, such as warm phases, caused population bottlenecks. Although polar bears have persisted through previous warm phases, multiple human-mediated stressors (e.g., habitat conversion, persecution, and accumulation of toxic substances in the food chain) could magnify the impact of current climate change, posing a novel and likely profound threat to polar bear survival.

References and Notes

1. D. Schluter, Science 323, 737 (2009).

2. P. A. Larsen, M. R. Marchán-Rivadeneira, R. J. Baker, Proc. Natl. Acad. Sci. U.S.A. 107, 11447 (2010).
3. J. L. Blois, E. A. Hadly, Annu. Rev. Earth Planet. Sci. 37, 181 (2009).

4. J. C. Uyeda, T. F. Hansen, S. J. Arnold, J. Pienaar, Proc. Natl. Acad. Sci. U.S.A. 108, 15908 (2011).

5. G. J. Slater, B. Figueirido, L. Louis, P. Yang, B. Van Valkenburgh, PLoS ONE 5, e13870 (2010).

6. S. C. Amstrup, in Wild Mammals of North America: Biology, Management, and Conservation, G. A. Feldhamer et al., Eds. (Johns Hopkins Univ. Press, Baltimore, MD, 2003), pp. 587-610.

7. C. Lindqvist et al., Proc. Natl. Acad. Sci. U.S.A. 107, 5053 (2010).

8. J. Davison et al., Quat. Sci. Rev. 30, 418 (2011).

9. C. J. Edwards et al., Curr. Biol. 21, 1251 (2011).

10. B. Kurtén, Acta Zool. Fenn. 108, 1 (1964).

11. G. F. Shields, T. D. Kocher, Evolution 45, 218 (1991).

12. M. Pagès et al., Mol. Phylogenet. Evol. 47, 73 (2008).

13. S. Nakagome, J. Pecon-Slattery, R. Masuda, Mol. Biol. Evol. 25, 1344 (2008).

14. L. Yu, Q. W. Li, O. A. Ryder, Y. P. Zhang, Mol. Phylogenet. Evol. 32, 480 (2004).

15. D. J. Funk, K. E. Omland, Annu. Rev. Ecol. Evol. Syst. 34, 397 (2003).

16. J. Heled, A. J. Drummond, Mol. Biol. Evol. 27, 570 (2010).

17. Material and methods and BEAST and STRUCTURE input files are available as supplementary materials on Science Online.

18. J. A. Leonard, R. K. Wayne, A. Cooper, Proc. Natl. Acad. Sci. U.S.A. 97, 1651 (2000)

19. D. Paetkau et al., Mol. Ecol. 8, 1571 (1999).

20. R. M. Zink, G. F. Barrowclough, Mol. Ecol. 17, 2107 (2008).

21. A. J. Welch, A. A. Yoshida, R. C. Fleischer, Mol. Ecol. 20, 1364 (2011).

22. G. Hewitt, Nature 405, 907 (2000).

23. F. A. Perini, C. A. M. Russo, C. G. Schrago, J. Evol. Biol. 23, 311 (2010)

24. M. Pilot et al., Mol. Ecol. 15, 4533 (2006).

25. B. P. Kelly, A. Whiteley, D. Tallmon, Nature 468, 891 (2010).

26. M. Currat, M. Ruedi, R. J. Petit, L. Excoffier, Evolution 62, 1908 (2008).

27. A. L. Roca, N. Georgiadis, S. J. O'Brien, Nat. Genet. 37, 96 (2005)

28. J. M. Good et al., Mol. Ecol. 17, 1313 (2008).

29. J. K. Pritchard, M. Stephens, P. Donnelly, Genetics 155, 945 (2000).

30. P. Beerli, Bioinformatics 22, 341 (2006)

31. T. M. Anderson et al., Science 323, 1339 (2009).

32. L. E. Lisiecki, M. E. Raymo, Paleoceanography 20, PA1003 (2005)

Acknowledgments: The study was supported by Hesse's LOEWE, Landes-Offensive zur Entwicklung Wissenschaftlich-ökonomischer Exzellenz. We thank E. W. Born, H.-G. Eiken, C. Frosch, S. Hagen, A. Kopatz, C. Nowak, M. Onucsán, M. Pfenninger, K. Skírnisson, F. Zachos, and P. Beerli for providing samples and for discussions. Obtained sequences have been deposited in the EMBL database (accession nos. HE657192 to HE657234 and HE657776 to HE658979). The authors declare no competing financial interests.

Supplementary Materials

www.sciencemag.org/cgi/content/full/336/6079/344/DC1 Materials and Methods

Figs. S1 to S5

Tables S1 to S5

References (33-61)

Supplemental Files S1 and S2 